Ainsi, dans bien des cas, les petites chaudières électricues peuvent-elles èlre employées avantageusement même lorsque le courant est produit à la vapeur. Ces chaudières sont surtout intéressantes lorsque, grâce à une accumulation facile à oblenir, il est possible de ne les faire fonclionner que pendant les heures de faible charge, la nuit surtout. C'est actuellement lo seul moyen vraiment pratique de combler les heures creuses des usines génératrices d'électricité.

\section{CONCLUSIONS}

Les chaudières életriques, dont l'emploi dans l'industrie date surtout de la fin de la guerre, sont appelées à se répandre de plus en plus, car ce sont actuellement les apparels les mieux adaplés pour utiliser les importants résidus d'énergie des chutes d'cau. Elles sonl doublement intéressantes en permettant de réaliser une économie de combustible très appréciable et en procurant ąux Sociétés Hydro-électriques un supplément de recette intéressant.

Pour les petites pulssances elles ont un très grand avenr. Les qualités de commodité cl de proprelé, qui les font tant apprécier par ceux qui les emploient, rendent en effet leur usage possible unême avec du courant à un prix assez élevé comme celui des centrales à vapeur. Les Sociélés de Distribution d'énergie commencent d'ailleurs à comprendre que, pour augmenter leur coefficienl d'utilisation, elles ont un grand intérêt à développer le plus possible l'emploi des chaudières à accumulation.

\title{
Application du Régulateur à action rapide aux Machines à Papier.
}

\author{
Par V. SYLVESTRE, Ingénieur A. M. et I. E. G.
}

\begin{abstract}
Les machines à papier modernes avec leur énorme produclion demandent non seulement un réglage de la vilcsse très étendu, mais il est de plus nécessaire que celle-ci ne soit pas influencée par des causes extêrieures el cela pour tous les régimes.

Les variations de vitesse se traduisent, en effet, par des inégalités dans l'épaisseur du papier qui en nécessitent la mise au rebut, ou bien par des déchirures ce qui donne lieu en fin de compte à des pertes sensibles, surtout aux grandes vitesses.

En outre, par suite de la hausse des matières premières, on a été amené à travailler,ces dernières annèes, des matières de qualité inférieure et ce fait augmente encore l'importance des avantages de la commande électrique. Seule celle-ci est en état de répondrè à toutes les exigences. D’où la tendance générale, bien justiłiée du reste, d'adopter la commande électrique dans toutes les fabriques de papier, non seulement lorsqu'il s'agit de nonvelles installations mais encore pour les fabriques existantes.

Etant donné l'importance de ce sujet, avant de décrire les applications du régulateur à action rapide, nous allons rappeler les différents systèmes de commande électrique employés aujourd'hui à la commande des machines à papier.
\end{abstract}

\section{CHOIX DU GENRE DE COURANT}

La plupart des machines dans les fabriques de papier travaillent à vitesso constante. D'après les expériences faites jusqu'ici, le courant triphasé s'est montré le mieux approprié à ces installations. En toul premier lien, ce sont les propriétés remarquables du moteur asynchrone triphasé qui, dans le choix du syslème, ont fait pencher la balance en favcur du courant triphasé.Il faut, il est vrai, transformex ce couranl pour l'alimentation du moteur de la machine à papier, mais il convient de remarquer qu'il an est de même dans les installations à courant continu, étant donné la nécessité de réaliser de grandes variations de vitesse, ainsi qu'une grande précision de réglage.

Etant domnć que les machines fonclionnant à vitesse constante sont celles qui, dans les fabriques de papier, consomment la plus grande quantité d'énergie, il semble opportun de profiter des avanlages des moteurs asynchrones et dadopter le courant triphasé pour l'ensemble de l'installation.

Comme l'emploi du courant triphasé dans les fabriques de papier semble devenir d'un usage courant, on a essayé d'utiliser également ces moleurs triphasés pour la commande de machines nécessitant un réglage de vitesse étendu.

Le moleur asynchrone, dont la vilesse se règle par insertion de résistances dans le circuit du rolor, ne peut convenir,étant donné, d'une part, les pertes élevées auxquelles donne lieu le mode de réglage et, d'autre part, la rclation à observer entre le couple et la vitesse. L'emploi d'un moleur triphasé à collecteurs permettrait,il est vrai, d'obtenir dans de larges limites un réglage cxempt de pertes, mais l'inconvénient résultant de l'instabilité de la vitesse n'en subsisterait pas moins, car le moteur triphasè à collectcur ayant une caractéristique série, les variations de charge ont pour effel de provoquer des variations de vitesse sensibles. En employant un moteur triphasé à collecteur ayant une caracléristique shunt, on arriverait, il est vrai, à diminuer l'influence des variations de charge sur la vitesse, mais les limites entre le quelles cet effet se fait sentir étant assez restreintes, il en résulte que ces moteurs ne permettraient pas non plus de réaliser un réglage de la vitesse dans de larges limites. Il ne reste donc qu une solulion, c'est de produire du courant triphasé et de le convertir en courant continu pour lalimentation des moteurs actionnant les machines à vitesse variable.

\section{COMMANDE ÉLECTRIQUE DES MACHINES A PAPIER}

Pour la commande de la partie à vitesse variable de la machine à papier, on emploie prestuue dans tous les cas, un moteur à courant conlinu excité en dérivation, dont on peut faire varier la vitesse dans de larges limites en agissant sur le champ et en faisant varier la tension du courant Lourni par la génératrice alimentant le moleur.

Si l'installation comporte plusieurs machines à papier dont la vilesse doit pouvoir varior dans le rapport de 1 à 3 ; par exemple si les sortes de papier diffèrent peu entre elles, comme c'est le cas pour les papiers courants destines à l'imprimerie, on peut se dispenser d'inslaller une genératrice par moteur. On prévoit, dans ce cas, l'alimentation de tous les moteurs par une commuta- 


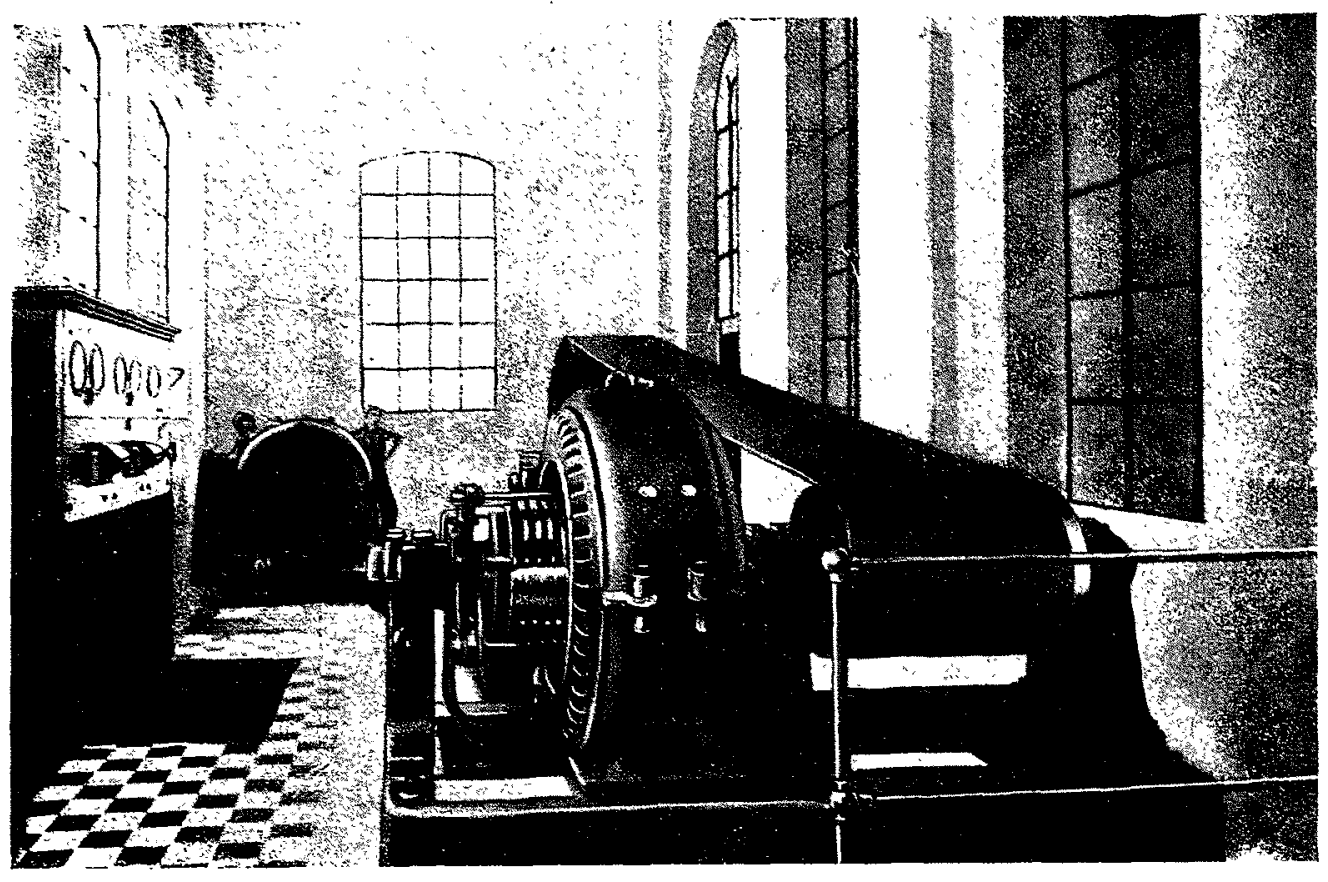

Fig. 55. - Commande de la partie à vitesse variable d'une machine à papier par un moteur à courant continu de 180 HP à vitesse réglable entre 150 et 980 tours pas minute au moren dn système Léonard et par variation du champ.

A l'arrière-plan un groupe moteur-générateur servant à alimenter les moteurs d'un rouleuse-bobineuse et d'une rouleuse-découpeuse, avec tableau de distribution.
La lonsion du courant d'alimenlation du moleur M peul varior daus le rapport de 1 à 5 ; les vitesses devées du moleur sonl oblenues par varialion de son champ magnélique.

Le courant nécessaice à l'oxcilation des deux machines est fommi par me excilatrice commune adossece au groupe: moleurgènérateur, à moins que l'usine nc dispose d'un réseatu à couranl conlinu.

linslallation comporte lous les appiareils al instruments nicessaires pour assurer la perfection el la securite du service.

Si on le desire, le demarrage da moteur 1) peut se faire au moyen d'un clemarrent adossé au moteur, portant linterruptentr du stator, une bobine de dedenchement en cas dexces ou de manque de comrant ot un ampèremedre. le disposilif de mise en court-circuil de's bangues el de relevage des balais esl verrouille mécaniquement à linterrupleur de slator el au rhéostal de démarrage, de manjere que la mise en marche on l'arrel du moteur peuvent se faire par la simple maneuye à la main d'un trice ou un groupe moteur-générateur unique si, bien entendu, l'installation ne comporte pas déjà un réseau à courant conlinu. Il est toutefois recommandable de maintenir la tension conslante en installant un régulateur à action rapide (1) qui permet de brancher sans précaution spéciale sur le même réseau dautres moteurs à vitesse variable ; par exemple, les moteurs de commande des enrouleuses el évenluellement ceux des calandres, sans qu'il y ail lieu de redouter que les à-coups provoqués par les arrèts et les démarrages fréquents de ces moleurs aient une influence sur la vitesse de la machine à papier. Grâce à ce régulateur, les moteurs des machines à papier sont alimentés sous une tension rigoureusement constante. Ainsi qu'il en a été dit plus haut, le réglage de la vitesse dans le rapport de 1 à 3 s'obtienl par variation du champ. Pour laver la toile métallique, lendre te feutre, etc., la vitesse peut encore être réduite par l'insertion du rhéostat de démarrage dans le circuit principal.

Cependant, dans la plupart des cas, on désire réaliser le réglage de vitesse dans des limites plus étendues et il e.t alors nécessaire d'alimenter chaque moteur par une génératrice spéciale.

Là, on utilise alors pour l'obtention du réglage de la viless" dans le rapport de 1 à 10, les propriélés du système Léonard.

\section{SYSTEME LEOONARD}

Partie électrique. - Le courant conlinu est produil par un groupe moleur-générateur, schéma figure $\mathrm{N}^{\circ} 50$, donl le moleur I) est branché sur le réseau triphasé, tandis que l'induit de la génératrice $G$ est relié directement au moteur $M$ de la machine à papier. L'excitalion de ce dernier est conslante, landis que l'excitation de la généralrice el par suilé, la lension du courant qu'elle produit, peuvent varier entre zéro et une valeur maxima. II esl ainsi possible de réaliser un démarrage très doux, puis d'obtenir toute la gamme des vitesses presque sans aucune perte d'energie.

(1) Voir Houille Blanche: Les Applications du Régulateur â action rapide (Janvier à Septembre 1922).

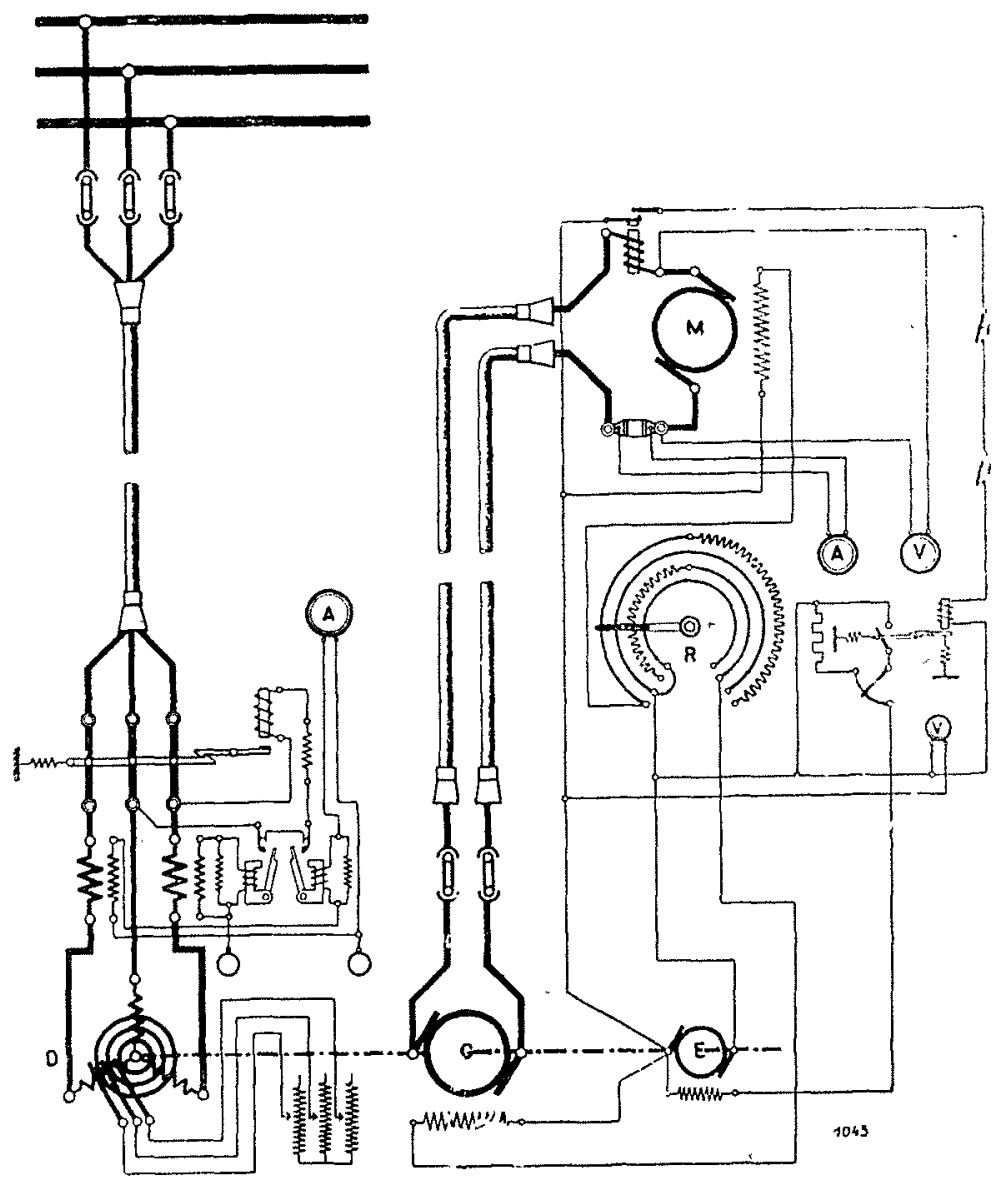

Fig. 56. - Schéma des commexions du système Léonard.

volant dans un sens ou dans un autre. Celle disposition convient surtout pour les installations où on ne dispose pas d'un personnel expérimenté, car elle rend impossible loute fausse mancouvre, tant au démarrage qu'à l'arrêt. Si cette disposition paraît superflue, il suffil alors de prévoir un colfret de mancuvre ordinaire el un rhéostat de démarrage séparé. Pour la généralrice el son exci- 
Latrice, on prévoil un lableau avec interrupleur, coupe-circuits, instruments de mesure, ansi qu'une résistance additionnelle pour l'excitatrice. Dans le coutoir de service de la machine à papier sont disposés le rhéostal de réglage $R$, ainsi qu'un ampèremétre et un voltmètre. Ce rhéostal $R$, figure $N^{\circ} 56$, sert au réglago des champs magnéliques de la génératrice el du moteur ; il esl manxuvré à la main par un volant muni d'une aiguille indiquant la vilesse correspondante de la machine à papier. La démarrage el le reglage de la vilesse du moleur principal M se font uniquement par variation de la tension pour les faibles el movennes vilesses. I a rotation du volant a pour effet, lorsque la pleine tension est alteinle, d'augmenter la vilesse du moleur par l'affaiblissement de son champ magnélique. Comme la tension d'alimentation reste constante, l'intensité du courant alugnente par suite de l'accroissement de Ia puissance. A cel effet, le moleur doil êlre construit pour l'intensité maximum du coumant correspondant à la puissance la plus élevés et, pour cetle raison, il peut dejà développer la puissance maximum à la vilesse qui correspond à la pleine tension el à la pleine excilation.

Si, par exemple, la puissance maxinum prescrite est de $100 \mathrm{HP}$ el que la vilesse doil pouvoir être réglée entre $100 \mathrm{el}$ 1000 lours par minute, savoir : entre 100 of 500 lours par variation de la lension dalimentation, et entre $500 \mathrm{el}$ 1000 lours par variation du champ, le moleur peut déjà développer $100 \mathrm{HP}$ en tournant à 500 tours. Pour une lonsion de $1 / \bar{j}$ de la lension normale correspondant à une vitesse de 100 Lours, le moleur développe encore 20 HP. Donc, lorsque la vilesse est réduite au $1 / 10$ de la vilesse maximum la puissance développéc par le moleur est encore de $1 / 5$ de la puissance exigée à la vilesse maximum. Il s'ensuil que le couple que le moleur est en mesure de fournir aux faibles vitesses est deux fois plus grand que celui qu’il développe aux vilesses supérieures. Celle considération conslitue un grand avantage lorsqu'il s'agil de la commande de machines à papier de construcI ion anciennc, car l'expérience a montré que le couple résistant de ees machines augmente quand la vilesse décroît. Toutefois, alle rend nécessaire l'emploi d'un moteur de type supérieur à celui qu'exigerail une machine travaillant sous couple constant.

Appareils de sécurité. -- I.es disposilifs de sécurilé du moleur Iriphasé sont cenx dont il a élé déjà question plus haul, à savoir le verrouillage entre le démarreur cl linterrupteur, ainsi que le relais pour le déclenchement à maximum et à tension nulle. Le relais à maximum déclenche automatiquement l'interruptear du moteur en cas de surcharge ; au contraire, en cas de manque? de lension, c'est la bobine à Lension nulle qui coupe le moteur du réseau. Cette disposilion a pour but d'éviler que l'interrupleur du moteur restc enclenché, alors que les bagues du rolor sont en court-circuil et que, par suite, le moteur soit en cet état pris sous courant au moment ou celui-ci sera rétabli par la slation centrale.

Des dispositions analogues peuvent être prévues pour les machines à courant conlinu. C'est ainsi que l'arrêt du moteur en cas d'excès de courant peut ètre obtenu par le déclenchement aulomatique d'un interrupleur inséré dans le circuit principal ou bien par la chule de la tension de l'excitalrice provoques par l'aclion d'un relais à maximum alimenté par le courant principal et insérant dans le circuit inducteur de l'excilatrice me résislance approprièe.

Ces disposilions permeltent d'obtenir facilement l'arrêt à distance de la machine à papier au moyen de boutons-poussoirs.

Si l'installation comporte un interrupteur autonatiqué à commande à distance, on peut prévoir un verrouillage électrique empêchant d'enclencher l'inlerrupteur lorsque le rhéostal de réglage ne se trouve pas dans la position de démarrage.

Partie mécanique. - Le moteur principal actionne générale-

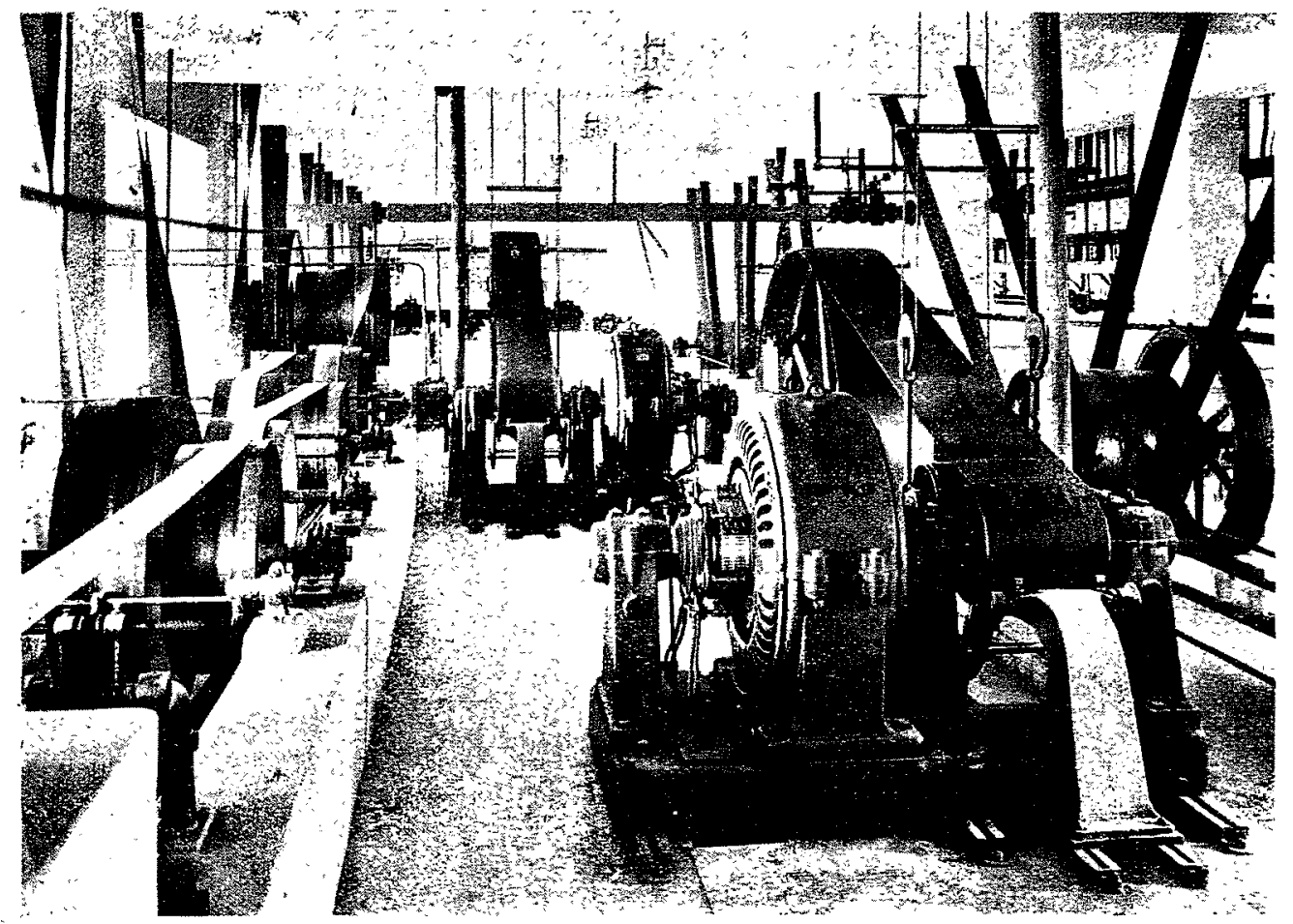

Hig: 57. - Noteurs à courant continu commandant deux machines à papier, produisant chacune par jour $15.000 \mathrm{~kg}$ de papier de $2.300 \mathrm{~mm}$. de largcur, à une vitesse de 120 mètres par minute.

ment la partic à vitesse variable de la machine à papier au moven

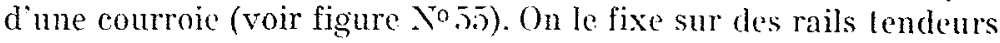
permellant de régler facilement la tension de la courroie. Icorsque Ic rapport de transmission des poulies est plus grand que 1 i $\overline{5}$, ou mème que 1 à $t$ et si la distance d'axe en axe des poulies ast très faible, l'emploi d'un enrouleur l,enix est à recommander.

La partie à vitesse constanle de la machine à papier est actionnée par un moteur spécial ne comportant pas de réglage de vitesse et qui est la plupart du temps un moteur asynchrone Iriphasé.

Mise en marche. - Le groupe moteur-générateur est tout d'abord mis en marche à vide, puis cn excite l'excitatrice jusqu’à ce qu'elle donne sa tension normale et enfin, on enclenche l'interrupteur. Fin agissant sur le rhéostal de réglage, on provoque le démarrage du moteur dont on règle la vitesse à la valeur voulue. Pour l'arrèt, on procède aux mèmes manceuvres, mais en sens inverse.

\section{SURVOLTAGE - DEVOLTAGE}

Si le réglage de la vilasse doit se faire dans un rapport supéricur de 1 à 10 ou bien s'il existe déjà un réseau à courant contiuu prévu pour alimenter plusieurs machines à papier, le système de 


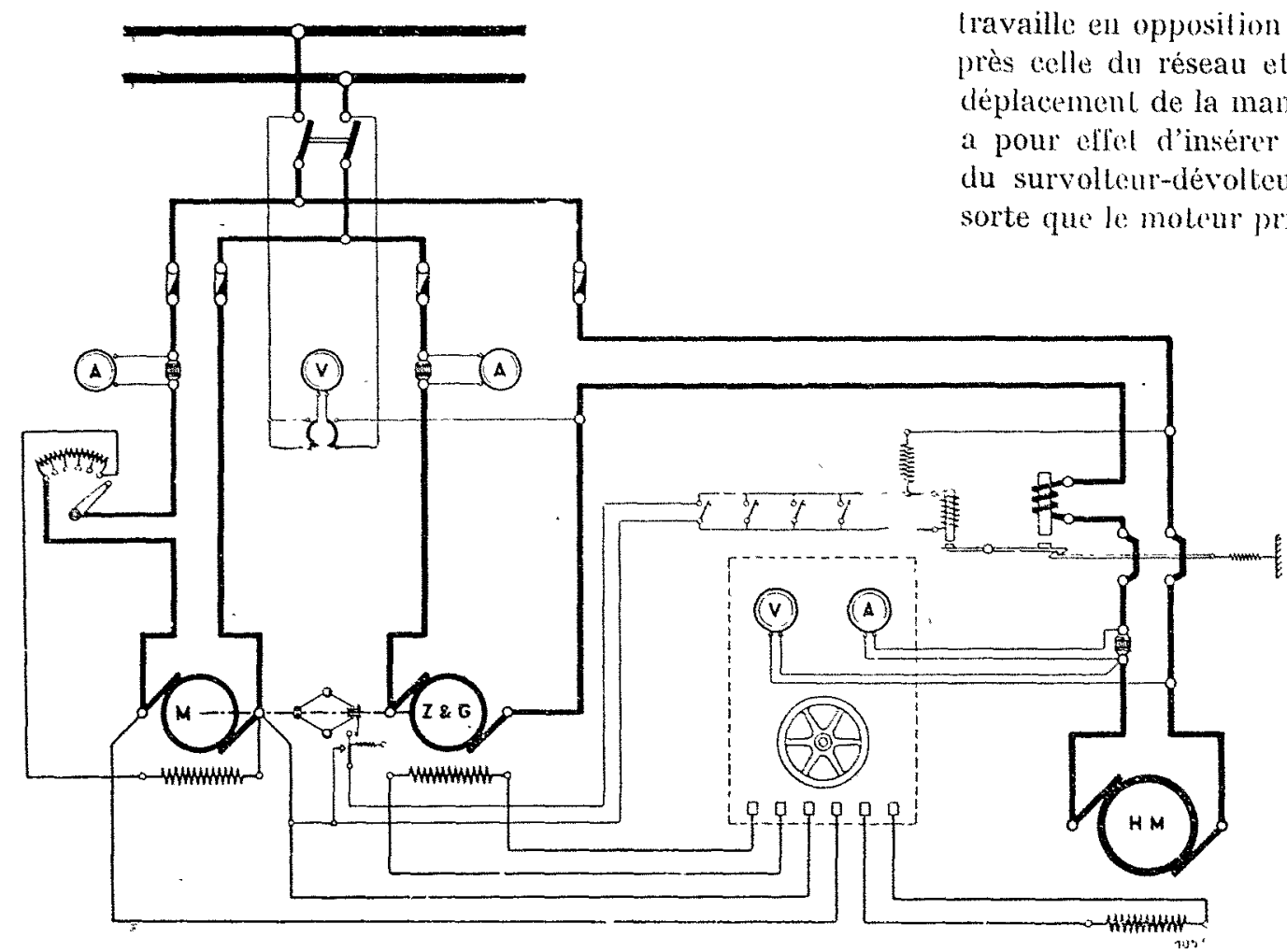

Fïg. 58. - Schéma de connexious : Règlage par groupe survolteur-dévolteur branclé sur un réseau à courant continu. réglage par survoltage-dévollage pourra être avantageusement employé.

Le principe de ce système consisle à faire varier la tensien au moyen d'un survolleur-dévolteur dont la tension se retranche (dévoltage) ou bien s'ajoute (survoltage) à celle du réseau. Comme la tension produite par le survolteur-dévolteur peut ètre égale à celle du réscau, il est possible de réaliser facilement le réglage de la tension entre une valeur nulle et une valeur double de la tension du réseau. D'autre part, comme le survolteur-dévolteur n'a à fournir qu'une tension égale ̀̀ la moitié de la tension maximum du moteur principal,cette machine n'est à prévoir que pour la demi-puissance de ce moteur.

Le survoltage-dévoltage peut s'effectuer de différentes manières :

A) L'installation comporte déjà une distribution à courant continu.

1. L La commande du survol-
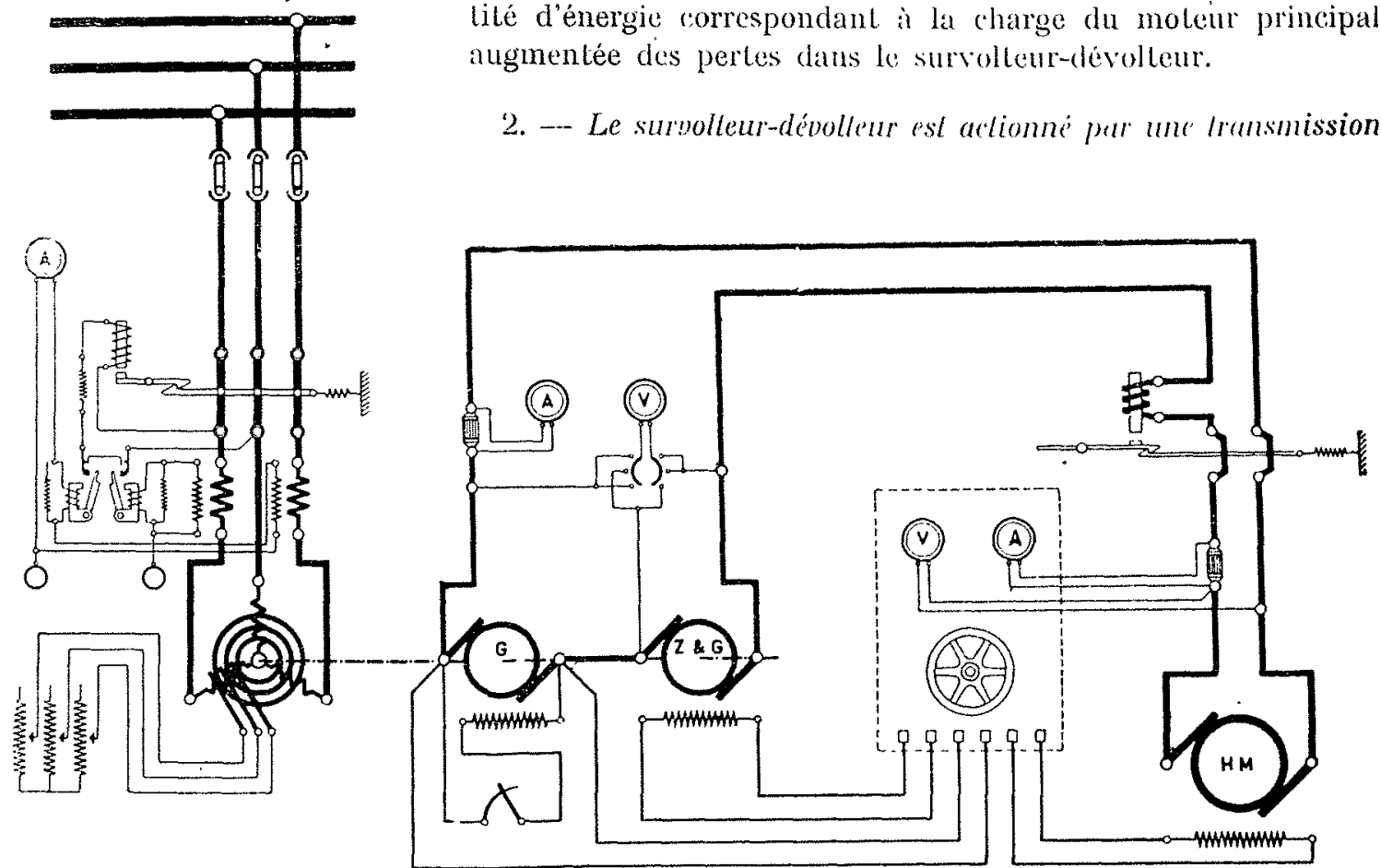

Fig. 59 - Schéma des connexions d'un groupe survolteur-dévolteur. I.e circuil a courant continu à tension constante est alimenté par la génératrice $G$.
Pendant la périzde de dévoltage, le survolteur-dévolleur fonctionne en réalité en moteur el son moleur do commande travaille au contraire en génératrice, débitanl du couranl sur ${ }^{1} \mathfrak{c}$ réseau. En conséquence. il n'est demandé au réseau que la quanlité d'énergie correspondant à la charge du moteir principal, augmentée des pertes daus le survoltenr-dévolteur.

\section{2. -- Le survolteur-dévollent est actionni pur unt Iransmission.}

teur-dévolteur, figure No 58 , se fait

au moyen d'un moteur à couranl continu. Le survolteur-dévolteur et son moteur sont accouplés directement et montés sur une plaque de base commune. Le réglage du moteur principal se fait au moyen d'un rhéostat-inverseur. Lorsque la manelle du rhéostal se trouve sur les premières touches, l'induit du survolteur-dévolteur qui est connecté en série avec l'induit du moteur principal
Ainsi que nous venons de le voir, pendant la période de dévoltage, lo survolteur-dévolteur travaille (n moteur ; daus le cas envisagé, il restiluera donc de l'énergie mécanique à la transmission. Celleci doil, bien entendu, lourner à vitesse constante et, par suite, cette dernière ne doil pas s'élever lorsqu'il y a récupération d'énergie. La puissance absorbée par la transmission doit donc 
Loujours être supéricure à la puissance maxima lournie par le survolteur et le moteur actionnant la transmission devra être muni d'un bon régulateur de vitesse. Telles sont les conditions nécessaires au bon fonctionnement de l'ensemble.

B) L'installation ne comporte pas encore de distribution à courant continu.

Si, seul, le moteur principal doil être alimenté par le réseau à courant continu, la génératrice et le survolteur-dévolteur peuvent être approximativement de même grandeur, et en travaillant ensemble, ils doivent pouvoir fournir l'énergie nécessaire au moleur principal. La génératrice doit, en outre, pouvoir fournir le courant d'excitation du survolteur-dévolteur el du moleur principal. Mais, si le réseau à courant conlinu est đestiné en outre à alimenter d'autres moteurs ou bien encore un circuit

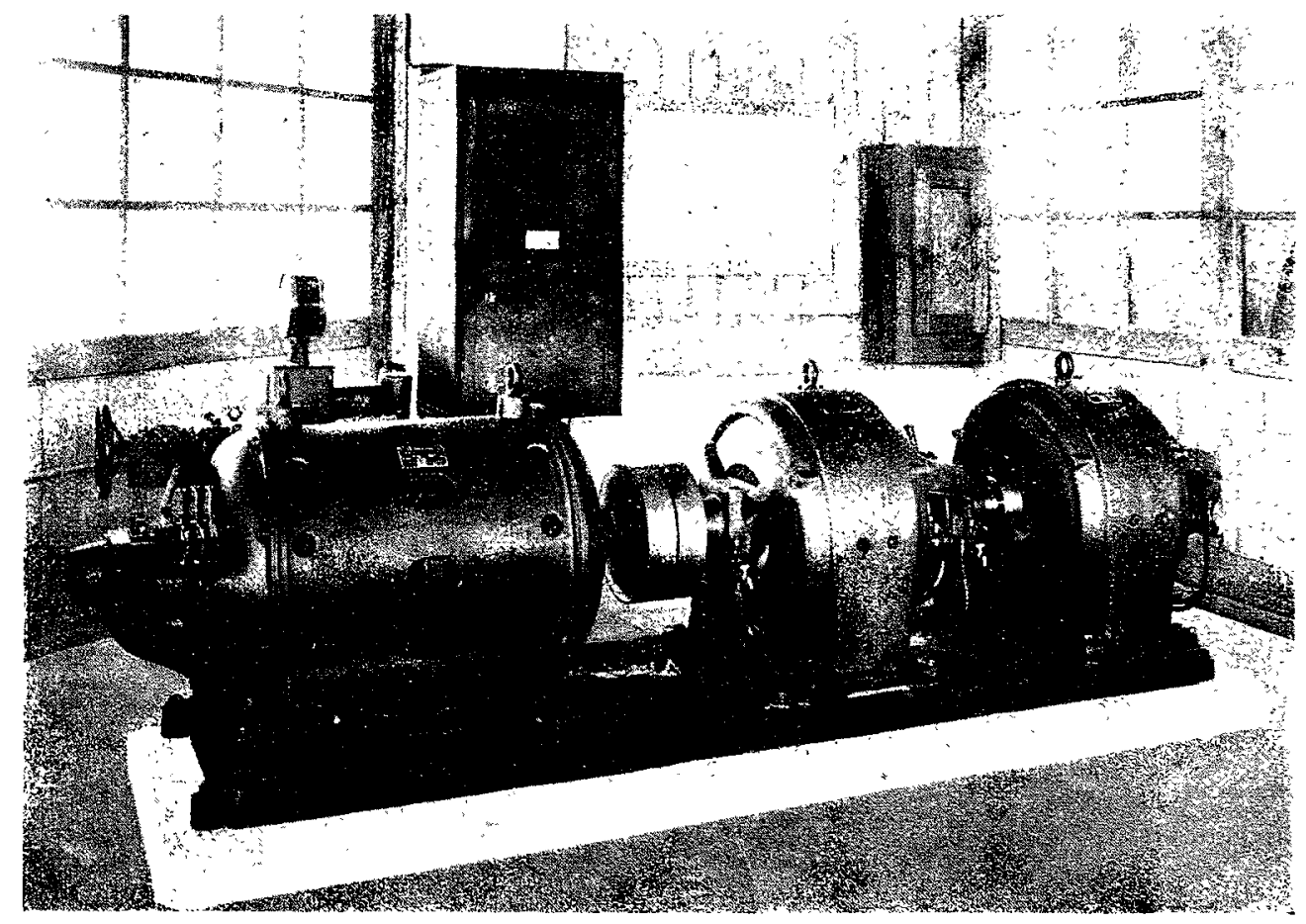

lFig. 60. - Survolteur-dévolteur pour le service d'un moteur de $120 \mathrm{HI}^{\circ}$ actionnant une machine à papier. de commande el que les fusibles de celui-ci sautent, le survolleurdévolteur fonctionnant alors en moteur non chargé, s'emballerasc immédiatement du lait de la faible valeur de l'intensité de son champ magnélique. Le même fait se produirait si le survolteurdévolteur élanl commandé par la courroie, celle-ci venait à se rompre ou mème simplement à tomber. L'interrupteur centrilinge a pour role de mettre hors circuit automatiquement le survolteur-dévolteur, dès que sa vitesse dépasse la valeur maximum admissible.

Les dispositifs que nous venons d'examiner suffisent dans la plupart des cas, grâce à un choix judicieux des machines el appareils, à maintenir au papier une vitesse de régime constante.

Cependant, si de forles variations de charge peuvent se produire à la station centrale électrique, résultant par exemple de l'inertie du régulateur d'une machine à vapeur, ou bien si la demande de vapeur aux chaudières se fait par à-coups, ou encore s'il se produit des variations de tension ou de fréquence des génératrices, ces divers changements de régime ont une répercussion inévitable sur le fonclionnement de la génératrice et du moteur de commande de la machine à papier el ont pour effel de provoquer des variations de vitesse du papier. On est alors obligé d'employer un dispositif maintenant rigoureusement constante la vitesse de régime du papier, et ceci indépendamment du fonctionnement de la machine à papier et des perturbations se produisant à la station centrale. Un réglage aussi précis ne peut ètre obtenu qu'avec un régulateur non seulement doué d'une grande sensibilité, mais possédant de plus une rapidité d'action lui permellant de parer instantanément aux variations venant à se produire.

Le régulateur de tension à action rapide satisfait entièrement à ces exigences. d'éclairage, on doit prévoir une génératrice plus puissante, capable de fournir le complément d'énergie.

Par contre, la puissance du survolteur-dévolteur doit toujours être égale à la moilié de celle du moteur principal. La génératrice et le sùrvolleur-dévolteur peuvent ètre actionnés, soit par un moteur unique, s'ils sont accouplés directement, soil au moyen de moteurs séparés ou bien encore par une transmission. La figure No 59 représente le schéma des connexions dans le cas de commande par un moteur électrique unique, et la figure No 60 représente le groupe des trois machines.

Appareils de sécurité. - Outre les appareils de sécurité nécessilés par le système Léonard, l'emploi du survolteur-dévolteur exige un dispositif spécial. Si le groupe n'est pas accouplè directement avec la génératrice produisant le courant à tension consLante, il doit être muni d'un interrupteur à force centriluge.

Comme on l'a déjà vu, il fonctionne comme moteur pendant la période de dévoltage. S'il est accouplé directement avec le moleur

\section{REGULATEUR AUTOMATIQUE DE TENSION APPLIQUE A}

\section{UN GROUPE LEONARD SERVANT A LA COMMANDE D'UNE MACHINE A CARTON.}

Le réglage automatique de la vilesse d'une machine à carton a été réalisé sur un groupe Léonard commandant un moteur à courant continu actionnant une machine a carton aux Papeteries Navarre.

L’ensemble du groupe Léonard est constilué par (voir schéma simplifie fig. 61):

$1^{\circ}$ Un moleur asynchrone triphasé $M_{1}$ accouplé par manchon élastique à

20 Une yénératrice G à courant continn de 138 A à tension variable de 20 à 260 volts, par varialıon de sun excitation.

$3^{\circ}$ Une excitatrice $\mathrm{E}$ fournissant le courant d'excitation nécessaire à la génératrice $\mathrm{G}$ el au moleur à couranl continu $\mathrm{M}_{2}$. 
4o Un moteur à courant continu $\mathrm{M}_{2}$ de $60 \mathrm{HP}$ a vitesse variable de 55 a 880 lours par variation de la lension d'alimentation de 20 a 260 volts, la tension dexcitation fournie par lexcitalrice E restant constante et égale à 200 rolls.
Pour pouvoir alimenter les bobines du régulateur à action rapide sous temsion conslante, quelle que soil la vilesse de la dynamo-lachymètre, on insère dans le circuil commun à la bobine? du régulaleur el a la tynamo-lachymetre une résistance $k_{2}$ dont le but est d'absorber l'exces de tension produil par la dyamo-lachymelre marchant à une vilesse phus élevé.

Le régulateur principal existant $h_{1}$ el le régulaleur $R_{2}$ sonl actionnés en mème temps. I a commande nécessaire pour ce dernier s'effectue soil par chaine de galle, soil par roue dentée, choix dépendant de la siluation locale. Le régulateur $R_{1}$ a pour but d'iliminer les differences inevilables dans la gradualion eles rhéostats $R_{1}$ el $R_{2}$. Le rhéostal R, serla lajuslage lin des vitesses.

Il faut remarquer que pour le réglage aulomatique,

A la plus faible vitesse la tension dexcilation de la génératrice G est de 20 volts, à la plus grande vitesse celte tension est de 132 volts. La vitesse de la toile de la machine a carton peut varier entre $2 \mathrm{~m}$. 50 et 25 mètres.

Le but du réglage est le suivant :

"Etant donnée une fabrication de carton pour " laquelle on doit marcher à une vilesse déterminée, le " conducteur de la machin agissant sur le rhéostal $R_{t}$ " de champ de la génératrice $\mathrm{G}$, amènera la machine à la " vitesse convenable. Cette manouvre étant effectuée, " le régulateur automalique doit maintenir la vitesse cons" tante à $\pm 1 \%$ près, quelle que soit la cause de la varia"tion de régime."

Ce problème a été résolu à l'aide d'un régulateur aulomatique et une dynamo tachymètre de la façon suivante (voir schéma figure No 62) :

Une petile dynamo, dénommée dynamo-lachymètre (6), est actionnée par le moteur principal $\mathrm{M}_{2}$ de la machine à carton. La tension de cette dynamo influence le régulateur à action rapide (7), qui fonctionne comme un régulateur de tension. Si la vitesse du moteur principal varie, la tension de la dynamo-tachymètre varie égalemenl. Le régulateur à aclion rapide entre en aclion inslantanément et ramène la vitesse à sa valeur initiale. A cel effet, le régulateur à action rapide influence le champ de la génératrice Léonard. Le régulateur fail varier le champ d'une excilatrice auxiliaire (4) qui fonctionne comme survolteur-dévolleur par rapport à la tension d'excitation des bobines de la dynamo Léonard. Si, par exemple, la vitesse du moleur principal diminue, la tension de la dinamo $f$ diminue aussi. Le régulateur à aclion rapide entre en action el augmente la tension d'excitation de la génératrice (6) el, par conséquent, la tension d'induit du molcur $\left(\mathrm{M}_{2}\right)$ et en même temps sa vilesse.

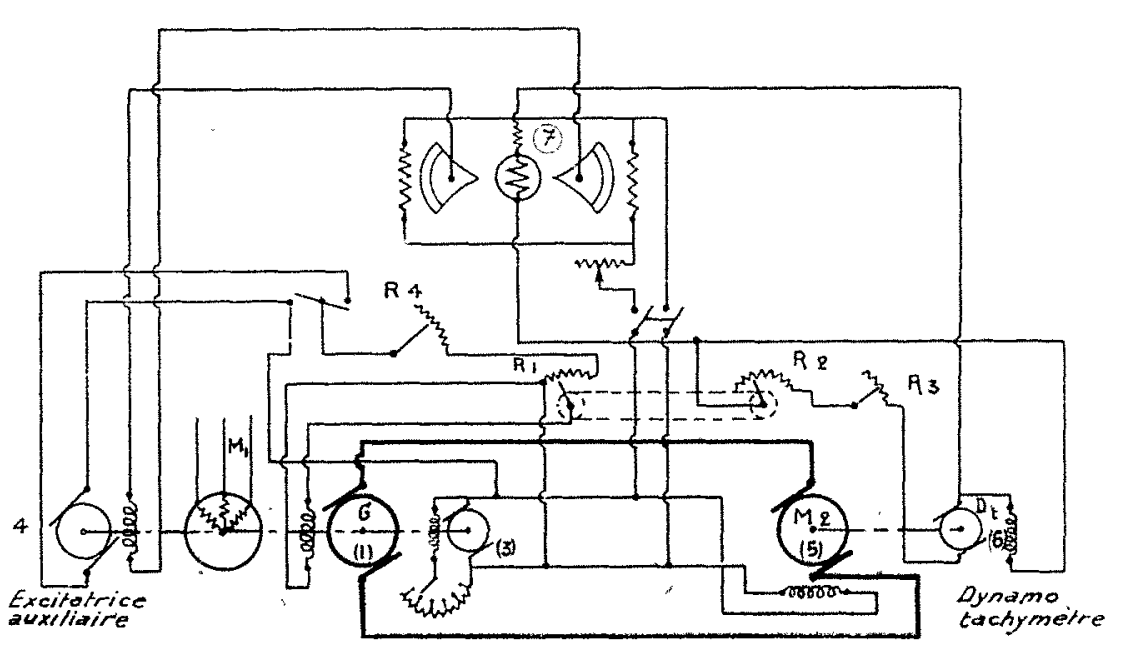

Fig. 62 .

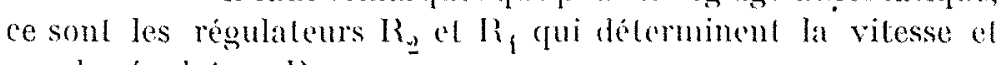
non le régulateur $l_{1}$.

Ce dispositif permel de passer du reglage a main au reglage 